\title{
The isentropic expansion energy of compressed and cryogenic hydrogen
}

\author{
G. Petitpas*, S. M. Aceves \\ Lawrence Livermore National Laboratory, \\ 7000 East Avenue, L-792, Livermore, CA 94550, USA \\ * Corresponding author: petitpas1@1lnl.gov
}

\begin{abstract}
Pressure is often perceived as the single most important parameter when considering the safety of a fluid storage system, for example the amount of energy released in the event of a sudden failure (burst energy). In this paper, we investigate the role of temperature as another degree of freedom for minimizing the burst energy. Results are first presented for ideal gases, for which the relationship between burst energy as a function of initial and final volumes, temperature and pressures can be expressed analytically. Similar analysis is then derived for the specific case of $\mathrm{H}_{2}$ using real gas equations of state. Assuming the expansion is isentropic, which holds for an adiabatic and sudden release as in a burst, it is shown that the energy released during a sudden burst is a weak function of pressure, revealing that the effect of pressure is negligible beyond a certain value ( 100 bar); whereas the burst energy is a linear function of temperature. This suggests that temperature controls the burst energy in a much greater way. This analysis is carried out in the frame of onboard $\mathrm{H}_{2}$ storage systems, for which it is shown that the use of cryogenic temperature for $\mathrm{H}_{2}$ vehicles, where risks of collision and impact on the surroundings are high, appears as a safety feature since burst energy is up to 18 times less than room temperature, high pressure storage.
\end{abstract}

\section{Introduction}

The very low density of hydrogen $\left(\mathrm{H}_{2}\right)$ at atmospheric conditions makes it difficult to store onboard a vehicle. Different technologies such as materials-based (adsorption or absorption), room temperature high pressure (350 to 700 bar [1]), or cryogenic storage at low (6 bar [2]) or high (350 bar [3]) pressure have been developed in order to store enough hydrogen within a reasonable weight and volume. These storage modes are characterized by thermodynamic states far away from equilibrium (e.g. atmospheric conditions) and are thus prone to release energy when hydrogen escapes the storage system.

We here investigate how both pressure and temperature affect the released expansion energy in case of sudden failure of a high-pressure storage system. In such a case, hydrogen is released to the atmosphere within a short time (seconds to minutes [4]), so that its expansion is assumed to be isentropic. Only calculations of total sudden released energy to atmospheric pressure are presented here (thermal equilibrium would take much longer to achieve). More detailed calculations on how expansion happens as a function of time for a cryogenic high pressure storage system can be found in [4]. 
Expansion energy calculations are shown first for ideal then for real gases, with an emphasis on the role of temperature.

\section{Expansion energy of an ideal gas with constant specific heat}

The expansion of an ideal gas with constant specific heats is described in this section. The ideal gas assumption becomes less and less valid as the initial conditions approach high pressures but is used here to simplify the analysis. Next section will conduct similar analysis using real gas equations of state.

The expansion phenomenon is identical to the gas expansion stroke in a piston engine. Assuming isentropic expansion, any thermodynamic state during the process can be computed from [6]:

$$
P V^{\gamma}=P_{0} V_{0}^{\gamma}
$$

Where $\gamma$ is the specific heat ratio $\left(\gamma=c_{p} / c_{v}=5 / 3\right.$ for monatomic and $\gamma=7 / 5$ for diatomic gases). As a result, the final volume for a release from a pressure $\mathrm{P}_{0}$ (in bar) to atmosphere $\left(\mathrm{P}_{1}=1\right.$ bar) is:

$$
V_{1}=P_{0}{ }^{1 / \gamma} V_{0}=P_{0}{ }^{(1-\gamma / \gamma)} R T_{0}
$$

At constant initial temperature, the final volume is a weak inverse function of pressure. Figure 1 shows the final volume $\mathrm{V}_{1}$ and temperature $\mathrm{T}_{1}$ for a vessel containing a diatomic molecule $(\gamma=7 / 5)$ from $T_{0}=300 \mathrm{~K}$ and various initial pressures $P_{0}$. Counterintuitively, the final volume of the expanded gas is actually lower for a higher initial pressure: high pressure $\mathrm{H}_{2}$ cools more and therefore expands less. 


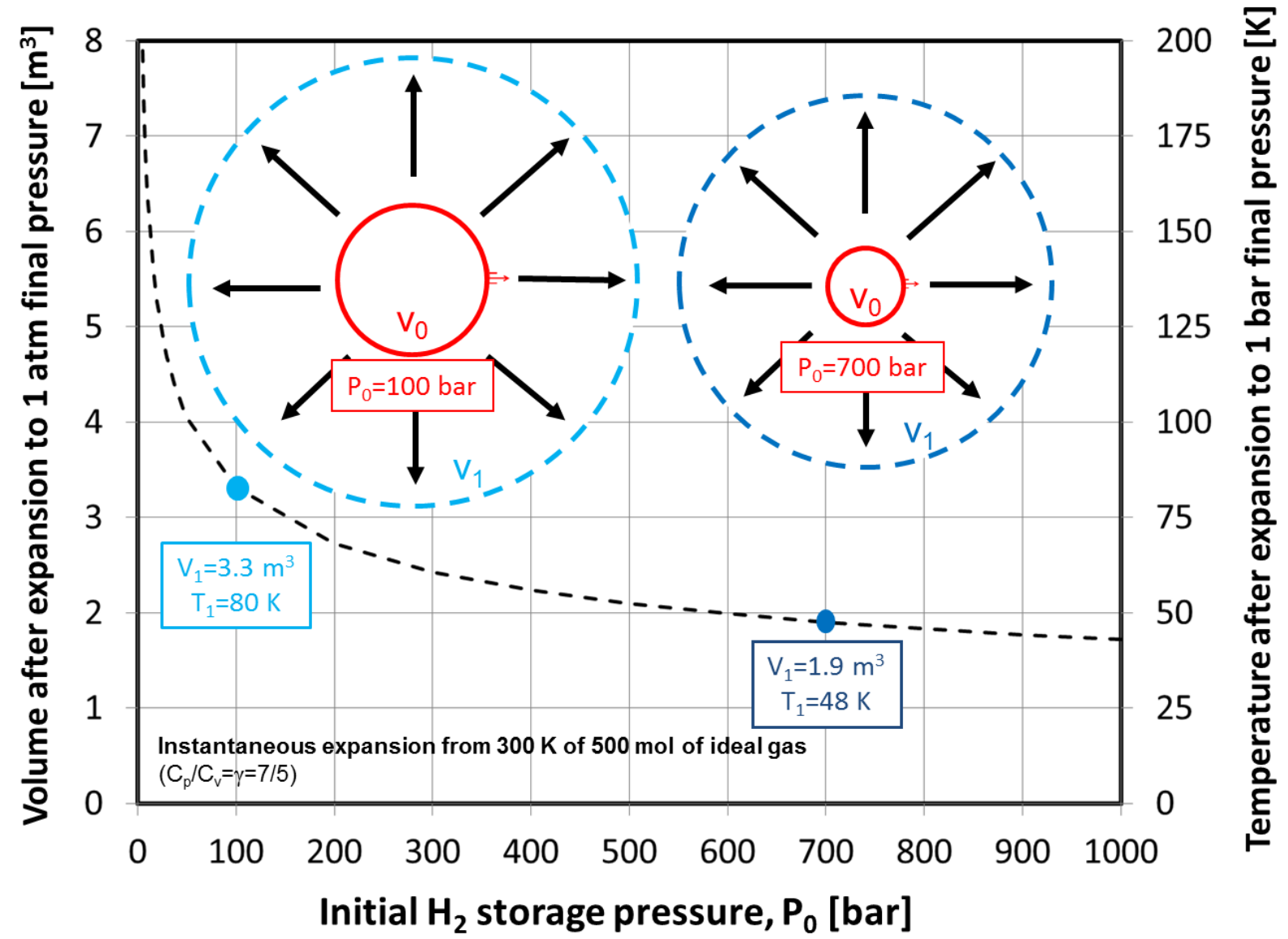

Figure 1. Final volume and temperature (both represented by the same dashed line) for an isentropic expansion of 500 moles (approximately equivalent to $1 \mathrm{~kg} \mathrm{H}_{2}$ ) of a diatomic ideal gas $(\gamma=7 / 5)$ from pressure $P_{0}$ and temperature $T_{0}=300 \mathrm{~K}$ to the atmosphere $\left(P_{1}=1 \mathrm{bar}\right)$.

The amount of energy released during gas expansion is equal to the expansion work and it is calculated as:

$$
E=\int P d V
$$

For ideal gas expansion, we substitute Equation (1) into Equation (3) and integrate from an initial thermodynamic state 0 at $\mathrm{P}_{0}, \mathrm{~V}_{0}, \mathrm{~T}_{0}$ to a final thermodynamic state 1 at $\mathrm{P}_{1}, \mathrm{~V}_{1}, \mathrm{~T}_{1}$ to obtain [7]:

$$
E=\frac{R T_{0}}{1-\gamma}\left(\left(\frac{V_{0}}{V_{1}}\right)^{\gamma-1}-1\right)
$$

Which is equivalent to: 


$$
E=\frac{R T_{0}}{1-\gamma}\left(1-\left(\frac{P_{1}}{P_{0}}\right)^{\frac{\gamma-1}{\gamma}}\right)
$$

Equations (4) and (5) directly show a linear relationship between initial temperature and expansion energy. We also see that for a large enough initial pressure $\mathrm{P}_{0}$, the term in parentheses approaches 1 and expansion energy is only a function of temperature:

$$
\lim _{P_{0} \rightarrow \infty} E=\frac{R T_{0}}{1-\gamma}
$$

Assuming constant values for $\mathrm{R}, \mathrm{T}_{0}, \gamma\left(7 / 5\right.$ for a diatomic molecule) and $\mathrm{P}_{1}$ ( 1 bar) for an ideal gas, the variation of the expansion energy as a function of the initial pressure $\mathrm{P}_{0}$ (in bar) can be mathematically described as:

$$
\begin{aligned}
& E\left(P_{0}\right)=\text { constant. } f\left(P_{0}\right) \\
& \text { with } f\left(P_{0}\right)=1-\frac{1}{P_{0}^{0.28}}
\end{aligned}
$$

We have $f(100$ bar $)=0.72$ and $f(1000$ bar $)=0.85$, hence expansion energy only increases $15 \%$ as the initial pressure increases from 100 to 1000 bar. Similarly to the variation of $\mathrm{V}_{1}$ with $\mathrm{P}_{0}$ (Figure 1), the isentropic expansion energy is a weak function of storage pressure for initial pressures beyond 100 bar. This is illustrated in Figure 2 for $300 \mathrm{~K}$ initial temperature and initial pressures up to 1000 bar $\left(\mathrm{P}_{1}=1\right.$ bar $)$. 


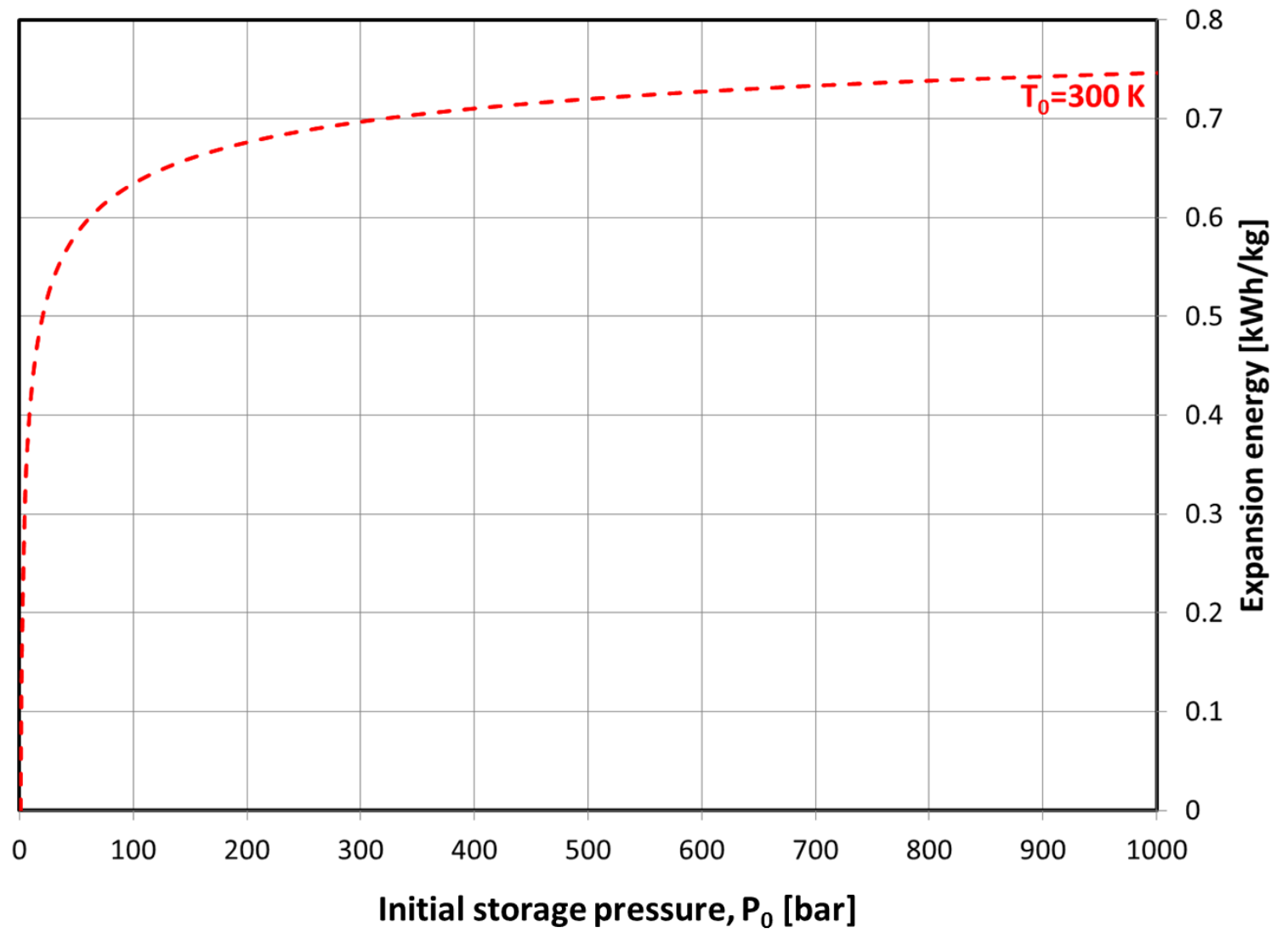

Figure 2. Expansion energy for isentropic expansion of a diatomic ideal gas with constant specific heats and specific heat ratio $\gamma=7 / 5$ initially at $300 \mathrm{~K}$ and pressure $\mathrm{P}_{0}$ to a final pressure $\mathrm{P}_{1}=1$ bar.

\section{Hydrogen expansion energy considering non-ideal equations of state}

The ideal gas law does not apply at the low temperatures and/or high pressures typical of today's hydrogen storage vessels. In this section, we analyze hydrogen's non-ideal gas behavior with the 32 term modified Benedict-Webb-Rubin [8] real gas equation of state using REFPROP [9]. Those equations have been shown to be the most accurate among the many available real gas equations of state [10].

For a real gas, we calculate the specific expansion energy as the isentropic expansion work,

$$
E=u_{P_{0}, T_{0}}-u_{P_{1}, s_{0}}
$$

Where $u_{P_{0}, T_{0}}$ is the internal energy at $\mathrm{P}_{0}$ and $\mathrm{T}_{0}$, and $u_{P_{1}, S_{0}}$ is the internal energy of hydrogen at 1 bar and the same entropy $\mathrm{s}_{\mathrm{o}}$ as the initial point $\mathrm{P}_{0}$ and $\mathrm{T}_{0}$ (for isentropic expansion, $\mathrm{s}_{1}=\mathrm{s}_{0}$ ).

At first, we compare the theoretical isentropic expansion energy of an ideal gas with constant specific heat vs. real hydrogen gas from room temperature $(300 \mathrm{~K})$. We observe (Figure 3$)$ that expansion energy of real $\mathrm{H}_{2}$ gas has even smaller variation above 100 bar than for the ideal gas: 
it only increases 5\% from 100 to 1000 bar (vs. 15\% for ideal gas). The slower increase in expansion energy vs. pressure is caused by the decreasing $\mathrm{H}_{2}$ specific heat (and therefore higher $\gamma$ ) as the $\mathrm{H}_{2}$ cools down during expansion (Figure 3 inset). As a consequence, the real gas cools more and expands less: the final expansion temperatures $\mathrm{T}_{1}$ (indicated on the red curves in Figure 3 ) are about $20 \mathrm{~K}$ colder for the real gas.

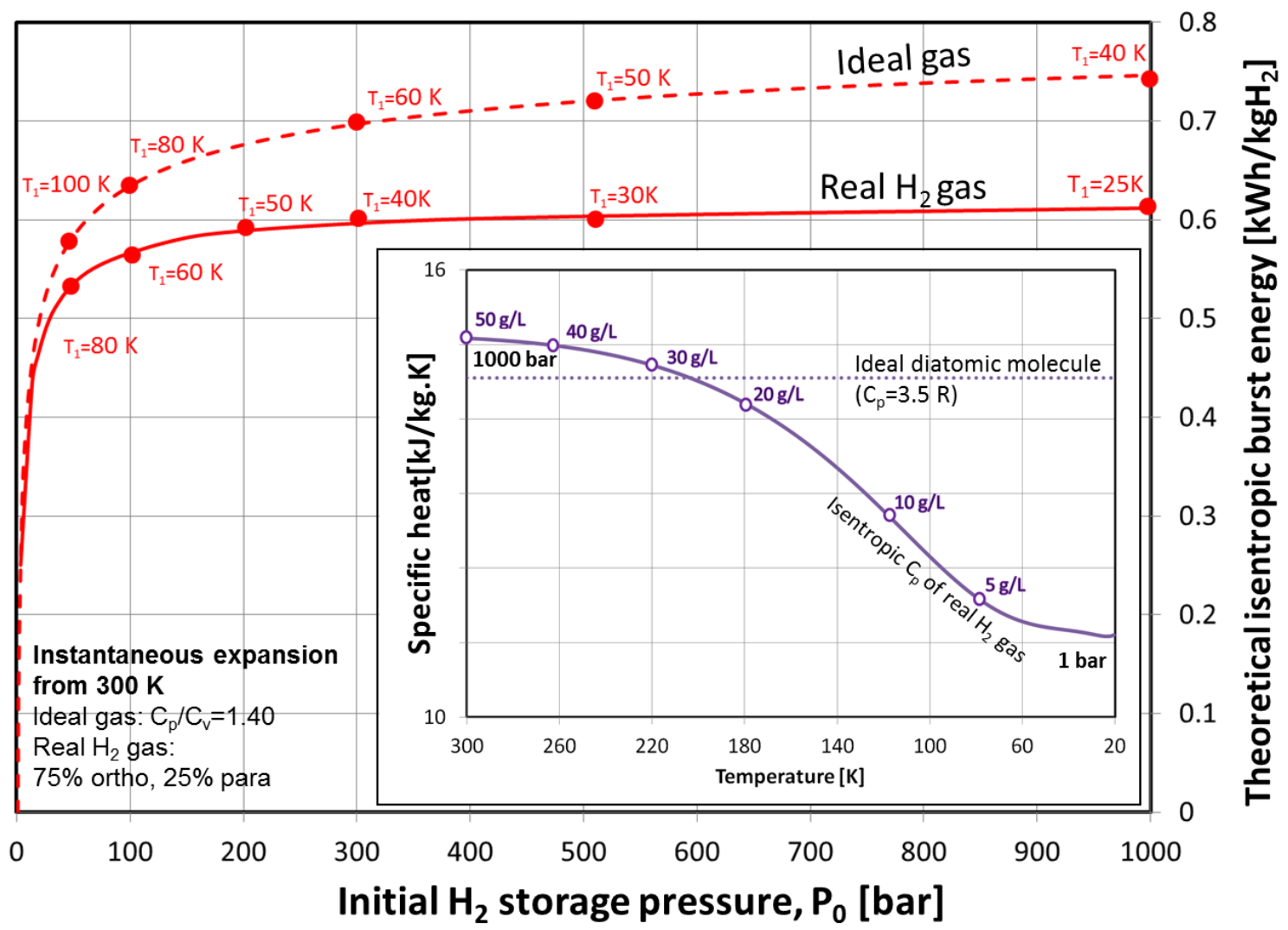

Figure 3. Theoretical isentropic expansion energy to atmospheric pressure $\left(\mathrm{P}_{1}=1 \mathrm{bar}\right)$ as a function of initial pressure $\mathrm{P}_{0}$ for an ideal diatomic gas with constant specific heats and for real $\mathrm{H}_{2}$ gas. Final expansion temperatures $\mathrm{T}_{1}$ are also shown for several initial expansion pressures. The inset shows specific heat at constant pressure vs. temperature for both the ideal diatomic molecule and the real $\mathrm{H}_{2}$ gas as they expand from 1000 bar and $300 \mathrm{~K}$.

We next consider expansion from cryogenic temperatures. Figure 4 shows isobaric (20 bar to 1000 bar) and isothermal $\left(50 \mathrm{~K}\right.$ and $300 \mathrm{~K}$ ) lines of $\mathrm{H}_{2}$ density vs. internal energy. Two isentropic lines are also represented: one starting from $50 \mathrm{~K}$ and $1000 \mathrm{bar}$, and the second from $300 \mathrm{~K}$ and 1000 bar. These dotted lines illustrate the sequence of thermodynamic states followed during isentropic expansion and are the graphical equivalent to Equation (1) for non-ideal $\mathrm{H}_{2}$. The reduced energy of cryogenic expansion becomes obvious, as the initial internal energy at low temperature is much lower than at ambient temperature. The expansion energy is directly computed as a horizontal distance along the $\mathrm{x}$-axis, by subtracting the initial and final internal energies: $0.61 \mathrm{kWh} / \mathrm{kg}$ for $\mathrm{T}_{0}=300 \mathrm{~K}, \mathrm{P}_{0}=1000$ bar vs. $0.043 \mathrm{kWh} / \mathrm{kg}$ for $\mathrm{T}_{0}=50 \mathrm{~K}, \mathrm{P}_{0}=1000$ bar. 


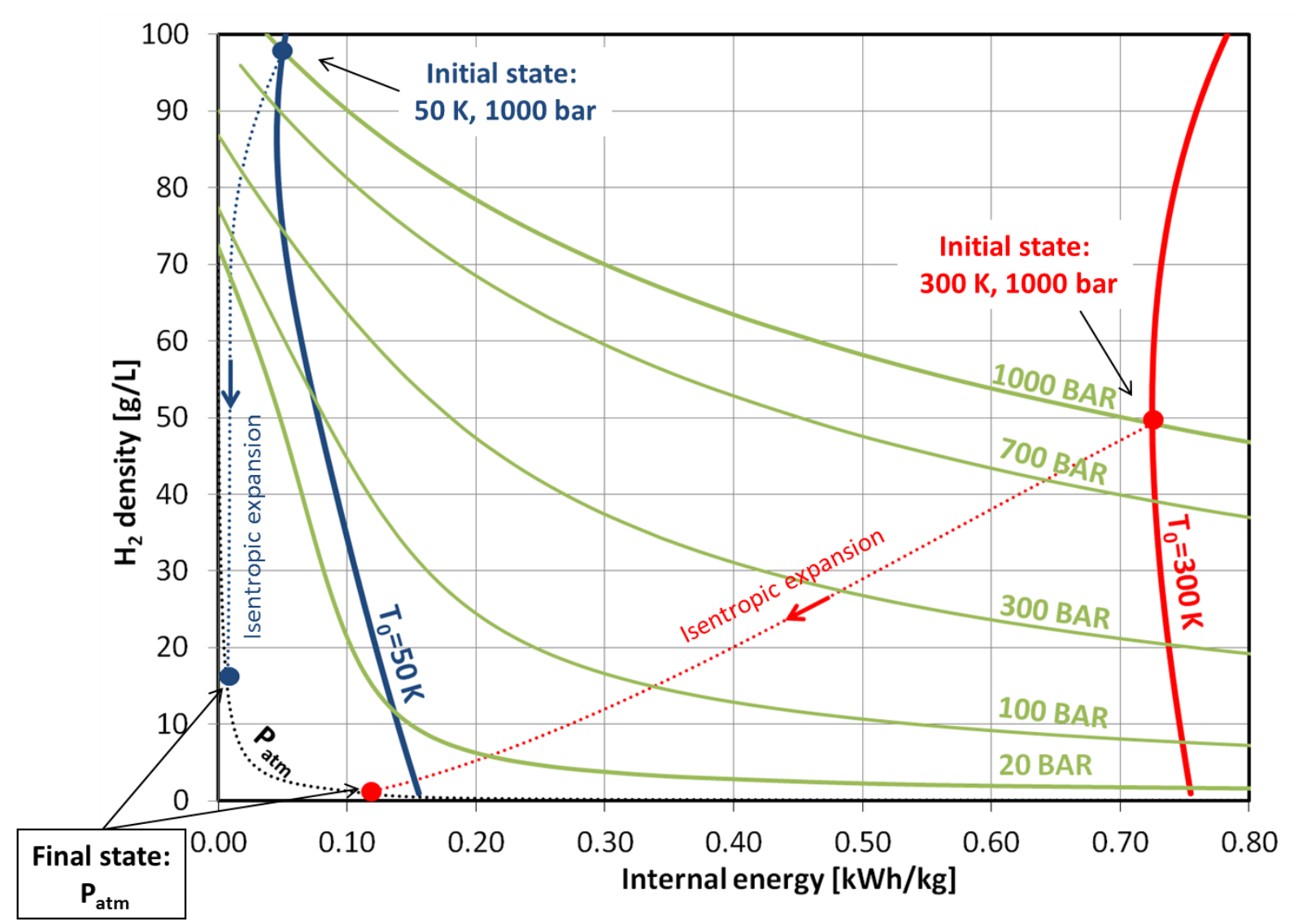

Figure 4. Internal energy and density paths for a $50 \mathrm{~K}, 1000$ bar and for a $300 \mathrm{~K}, 1000$ bar isentropic expansion to atmospheric pressure.

Similar analysis can be conducted for the whole range of initial pressures, and the results of Figure 5 indicate that cold temperature storage $\left(\mathrm{T}_{0}=50 \mathrm{~K}\right)$ reduces expansion energy by a factor of 18 compared to expansion from $300 \mathrm{~K}$ at pressure typical of today's $\mathrm{H}_{2}$ storage vessels (350700 bar).

Figure 5 also indicates the typical working conditions of other advanced $\mathrm{H}_{2}$ storage technologies that are being researched, such as metal hydride [1] and adsorbent [10], and the corresponding theoretical isentropic burst energy of the $\mathrm{H}_{2}$ molecules that are in their gaseous state at those conditions. In the event of a sudden release, only the molecules that are moving freely in the storage tank would participate in the expansion event. 


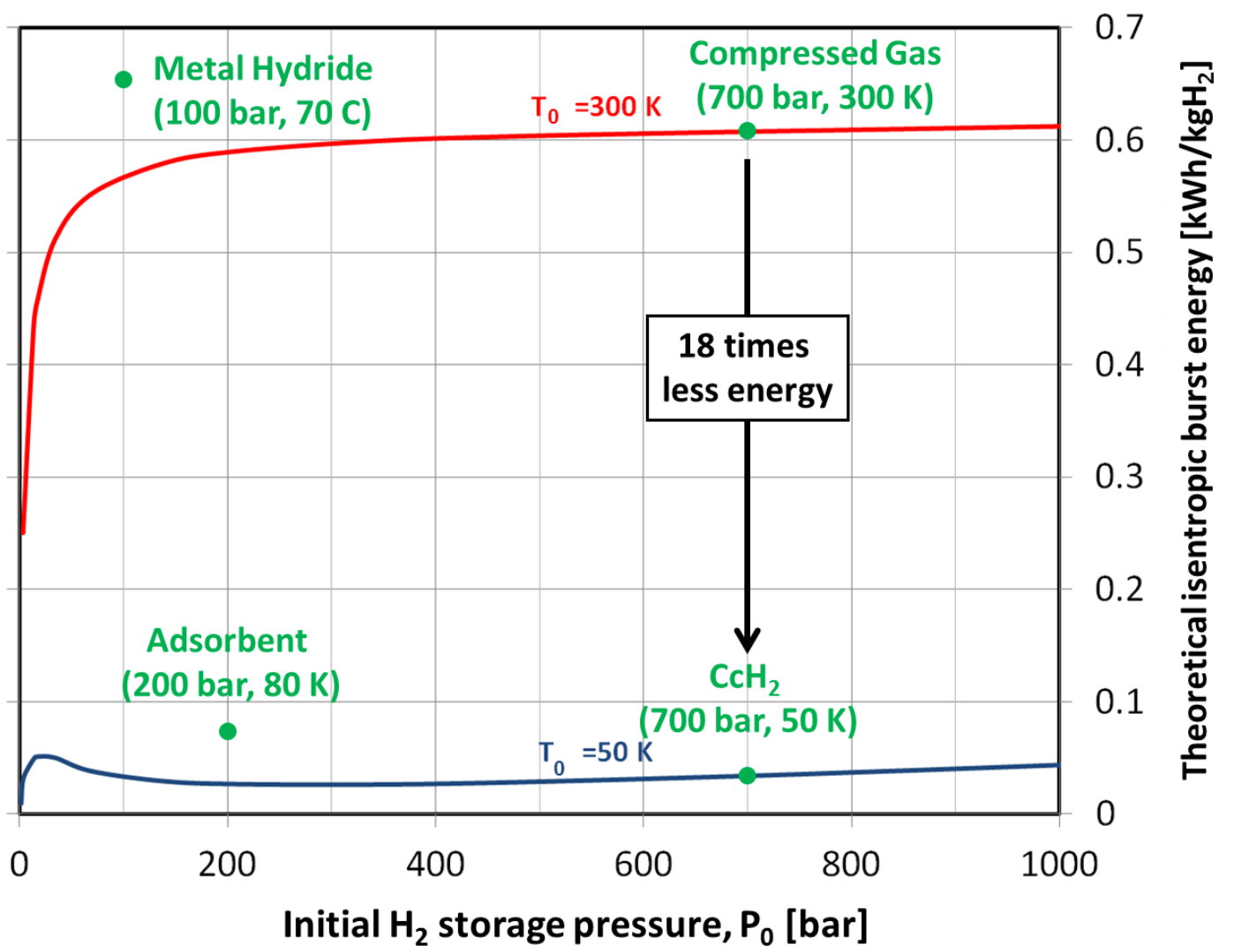

Figure 5. Effect of initial temperature $\left(\mathrm{T}_{0}=50\right.$ and $\left.300 \mathrm{~K}\right)$ on isentropic expansion energy as a function of storage pressure. Typical operating conditions of four different onboard storage technologies (metal hydride [1], compressed gas, adsorbent [1],[11] and cryogenic pressurized storage [12]) are also shown on the figure.

\section{Conclusions}

Pressure is often perceived as the most important parameter for safety ("the higher the pressure, the higher the risk"). In this paper, we describe how temperature is frequently the parameter that determines expansion energy, and therefore has key importance in the design of safer hydrogen storage systems.

Within the ideal gas assumption, it is first shown that the final expansion volume is inversely proportional to the initial gas pressure. This unexpected result happens because the higherpressure gas cools more. For a given initial temperature and final pressure, the expansion volume varies as $P_{0}^{1 / \gamma^{-1}}$; which directly translates into similar dependency for the burst energy calculation. In terms of physical behavior, this means that the burst energy is roughly linear with pressure up to 100 bar, and then undergoes a much weaker increase: the burst energy varies only by $15 \%$ when pressure is increased from 100 to 1000 bar. On the other hand, the initial temperature is a multiplicative factor in the burst energy calculation, hence a much stronger relationship: for any given pressure, the burst energy is doubled when the initial temperature doubles. These remarks hold true for any ideal gas. 
The analysis was then conducted for real gas $\mathrm{H}_{2}$, an assumption that is more accurate for high pressure and low temperature initial conditions. Although similar behavior holds, an even weaker dependence of burst energy with pressure and a stronger influence of the temperature are observed. An increase of the burst energy of only 5\% when increasing the pressure from 100 to 1000 bar is observed for an initial temperature of $300 \mathrm{~K}\left(+0.1 \%\right.$ for $\left.\mathrm{T}_{0}=50 \mathrm{~K}\right)$, while the burst energy can be up to 18 times lower when reducing the temperature from $300 \mathrm{~K}$ to $50 \mathrm{~K}$ (vs. a factor of 6 reduction for ideal gases).

We can conclude that in order to greatly improve the safety of $\mathrm{H}_{2}$ storage, lowering the temperature is much more efficient than lowering its pressure. Cryogenic storage, even when combined with high pressures, offers unprecedented safety in terms of energy released per $\mathrm{kg}$ of stored $\mathrm{H}_{2}$ in the event of a sudden failure. Cryogenic storage, even at very high pressure such as 1000 bar and above, has a lower burst energy than, for example, room temperature storage at 5 bar. Lowering pressure should not be considered a safety enhancement if temperature remains the same.

\section{Acknowledgments}

This project was funded by DOE, Office of Fuel Cell Technologies, Ned Stetson, Erika Sutherland, and Jason Marcinkoski, Technology Development Managers. This work performed under the auspices of the U.S. Department of Energy by Lawrence Livermore National Laboratory under Contract DE-AC52-07NA27344.

\section{References}

[1] Stetson N. An Overview of U.S. DOE's activities for Hydrogen Fuel Cell Technologies 2012, Materials Challenges in Alternative \& Renewable Energy (MCARE), Clearwater, FL.

http://ceramics.org/wp-content/uploads/2012/02/mcare12-hydrogen-fuel-cell-tech1.pdf (last accessed September 2014)

[2] Wallner T, Lohse-Busch H, Gurski S, Duoba M, Thiel W, Martin D, et al. Fuel economy and emissions evaluation of BMW Hydrogen 7 Mono-Fuel demonstration vehicles. International Journal of Hydrogen Energy 2008;33:7607-18. doi:10.1016/j.ijhydene.2008.08.067.

[3] Aceves SM, Espinosa-Loza F, Ledesma-Orozco E, Ross TO, Weisberg AH, Brunner TC, et al. High-density automotive hydrogen storage with cryogenic capable pressure vessels. International Journal of Hydrogen Energy 2010;35:1219-26. doi:10.1016/j.ijhydene.2009.11.069.

[4] Petitpas G, Aceves SM. Modeling of sudden hydrogen expansion from cryogenic pressure vessel failure. International Journal of Hydrogen Energy 2013;38:8190-8. doi:10.1016/j.ijhydene.2012.03.166.

[6] Wylen RES 'Gordon JV. Fundamentals of Classical Thermodynamics. 2nd ed. John Wiley \& Sons; 1985.

[7] Mannan S, Lees FP. Lee's Loss Prevention in the Process Industries: Hazard Identification, Assessment, and Control. vol. 3. Elsevier; 2005. 
[8] Younglove BA. Thermophysical Properties of Fluids. 1. Argon, Ethylene, Parahydrogen, Nitrogen, Nitrogen Trifluoride, and Oxygen. vol. 11. American Institute of Physics; 1982.

[9] Lemmon EW, Huber ML, McLinden MO. NIST Standard Reference Database 23: Reference Fluid Thermodynamic and Transport Properties-REFPROP 2007.

[10] Markert F, Melideo D, Baraldi D. Numerical analysis of accidental hydrogen releases from high pressure storage at low temperatures. International Journal of Hydrogen Energy 2014;39:7356-64. doi:10.1016/j.ijhydene.2014.02.166.

[11] Chakraborty A, Kumar S. Thermal management and desorption modeling of a cryoadsorbent hydrogen storage system. International Journal of Hydrogen Energy 2013;38:3973-86. doi:10.1016/j.ijhydene.2012.11.113.

[12] Aceves SM, Petitpas G, Espinosa-Loza F, Matthews MJ, Ledesma-Orozco E. Safe, long range, inexpensive and rapidly refuelable hydrogen vehicles with cryogenic pressure vessels. International Journal of Hydrogen Energy 2013;38:2480-9. doi:10.1016/j.ijhydene.2012.11.123. 